\title{
Multidisciplinary approach to the diagnosis and treatment of patients with potentially resectable colorectal cancer liver metastasis: results of a multicenter study
}

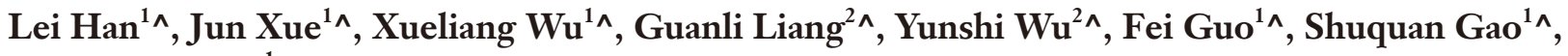 \\ Guangyuan $\operatorname{Sun}^{1 \wedge}$
}

${ }^{1}$ General Surgery Department, The First Affiliated Hospital of Hebei North University, Zhangjiakou, China; ${ }^{2}$ Graduate School, Hebei North University, Zhangjiakou, China

Contributions: (I) Conception and design: J Xue; (II) Administrative support: X Wu; (III) Provision of study materials or patients: L Han, G Liang; (IV) Collection and assembly of data: L Han, Y Wu, G Sun; (V) Data analysis and interpretation: L Han, F Guo, S Gao; (VI) Manuscript writing: All authors; (VII) Final approval of manuscript: All authors.

Correspondence to: Jun Xue, Xueliang Wu. General Surgery Department, The First Affiliated Hospital of Hebei North University, Zhangjiakou, China. Email: yfyxuejun@163.com; wuxl@hebeinu.edu.cn.

Background: This study sought to review colorectal cancer liver metastasis (CRLM) patients at multiple
centers to analyze the factors affecting the success of conversion therapy in patients whose CRLM was
initially evaluated as potentially resectable, to explore the effect of different treatment approaches on patient
survival, and to provide a scientific reference for clinical treatment of CRLM.
Methods: Fifty patients whose CRLM was initially evaluated as potentially resectable at 3 large Chinese
general hospitals were enrolled in this retrospective study. Statistical analyses were carried out on the general
data and pathological characteristic data to examine the clinical efficacy of the treatment approaches. The
factors affecting the success of conversion therapy were analyzed by logistic regression. Additionally, follow-
up appointments were conducted to examine survival, and survival curves were plotted using the Kaplan-
Meier estimator. The effect of different clinical and pathological characteristics on CRLM patients was
analyzed.

Results: Seventeen patients achieved no evidence of disease (NED) status through surgical resection/ ablation after undergoing conversion therapy. The multifactor analysis demonstrated that the number of liver metastases was the primary risk factor affecting the efficacy of conversion therapy $(\mathrm{P}<0.05)$. Survival analysis results showed statistically significant difference in overall survival (OS) between the NED group and the inconspicuous/progressive group $(\mathrm{P}<0.0001)$. Also, there was a statistically significant difference in the progression-free survival (PFS) between the NED group and the inconspicuous/progressive group $(\mathrm{P}<0.0001)$. Patients in the surgical resection group had better OS and PFS than those in the ablation group $(\mathrm{P}<0.0001$ and $\mathrm{P}<0.01$, respectively). The monofactor analysis demonstrated that the number and maximum diameter of liver metastases, serum Carcino-Embryonic Antigen (CEA) level, and BRAF V600E mutation status were factors affecting the OS of CRLM patients $(\mathrm{P}<0.05)$, of which BRAF V600E mutation was the primary determinant $(\mathrm{P}<0.05)$.

Conclusions: Among the patients whose CRLM was initially evaluated as unresectable, those who underwent surgical resection of the primary lesions and liver metastases after receiving conversion therapy had the best prognosis. Thus, a thorough evaluation should be conducted to determine the effect of and survival factors affecting conversion therapy in the treatment of liver metastases.

\footnotetext{
$\wedge$ ORCID: Xueliang Wu, 0000-0001-5883-6597; Lei Han, 0000-0002-2322-1055; Jun Xue, 0000-0002-5614-7859; Guanli Liang, 00000003-1049-5409; Yunshi Wu, 0000-0002-1596-5185; Fei Guo, 0000-0001-8414-5626; Shuquan Gao, 0000-0001-5231-3875; Guangyuan Sun, 0000-0002-5615-459X.
} 


\begin{abstract}
Keywords: Multidisciplinary collaboration; colorectal cancer liver metastasis; diagnosis and treatment; efficacy; prognosis
\end{abstract}

Submitted Dec 14, 2021. Accepted for publication Feb 21, 2022.

doi: $10.21037 / \mathrm{apm}-22-87$

View this article at: https://dx.doi.org/10.21037/apm-22-87

\section{Introduction}

The major target organs of distant metastases from colorectal cancer are the liver and lungs (1). Colorectal cancer liver metastasis (CRLM) is the most common cause of death in colorectal cancer patients. Approximately $15-25 \%$ of patients with confirmed colorectal cancer have synchronous liver metastases, and another $15-25 \%$ of patients will develop synchronous liver metastases following the radical resection of primary lesions. Among the latter patients, as the overwhelming majority of liver metastases are not initially suitable for radial (R0) resection, these patients must undergo a series of conversion therapies. Thus, the overall survival (OS) of patients with such lesions is short (2-4). According to relevant research, the median OS of patients with liver metastases who have received no treatment is only 6.9 months, and the 5 -year survival rate of patients with unresectable liver metastases is $<5 \%$. Conversely, if the liver metastases can be completely resected or if patients can achieve no evidence of disease (NED) status, their median OS is 35 months, and their 5 -year survival rate can be up to $30-57 \%(5,6)$.

Before 2018, China's guidelines for CRLM set R0 resection as the therapeutic goal for CRLM, requiring the complete resection of liver metastases and the surgical preservation of certain incisal edges. However, after 2018, the relevant consensus on diagnosis and treatment changed R0 resection to NED, which was extended to a larger CRLM patient population than the original $\mathrm{R} 0$ resection that was limited to a specific patient population $(7,8)$. The treatment of such CRLM patients should be conducted to be evaluated comprehensively and systematically under the guidance of a multidisciplinary team (MDT) which includes internal medicine, radiotherapy, interventional imaging (MRI, CT and ultrasound), pathology and other related disciplines. MDT collaborations should seek to develop individualized therapeutic goals and administer comprehensive therapies (e.g., surgical resection, genetic testing, chemotherapy, radiotherapy, target therapy, radiofrequency, microwave, and embolotherapy) to treat primary and metastatic lesions during one or several operations, thereby improving the overall surgical resection rate, ablation rate, and 5-year survival rate of CRLM patients.

Based on this concept, we invited three large regional general hospitals, including the Hebei Tumor Hospital and Tianjin Nankai Hospital, to retrospectively collect information on the incidence, diagnosis, and treatment of patients diagnosed with CRLM, with an emphasis on patients whose CRLM was initially evaluated as potentially resectable. The regimens and effects of conversion therapy were analyzed, and ablation therapy was added on the basis of previous surgical treatment, so as to expand the treatment indications of NED. Additionally, the survival of CRLM patients was analyzed based on survival follow-up results to provide a scientific basis for subsequent precision therapy and evaluation. We present the following article in accordance with the STROBE reporting checklist (available at https://apm.amegroups.com/article/view/10.21037/apm$22-87 / \mathrm{rc})$.

\section{Methods}

\section{Study design and study participants}

CRLM patients at the General Surgery Department of The First Affiliated Hospital of Hebei North University, No. 2 Surgery Department of Hebei Tumor Hospital, and No. 1 Gastrointestinal Surgery Department of Tianjin Hospital of ITCWM Nankai Hospital between October 1, 2017 and October 1, 2018 were enrolled in this retrospective study. The general data of the patients were recorded, including their age, gender, degree of tumor differentiation, tumor pathological type, tumor clinical stage (in accordance with the 8th edition of staging criteria by the American Joint Committee on Cancer) (9), chemotherapy response score (CRS), metastatic tumor diameter, carcinoembryonic antigen (CEA) level, the number of liver metastases, the surgery method, genetic testing results, and conversion therapy data. All procedures performed in this study involving human participants were in accordance with the 
Declaration of Helsinki (as revised in 2013). The study was approved by the ethics committees of The First Affiliated Hospital of Hebei North University (approval ID 2021013), Hebei Tumor Hospital (approval ID 2017kf386) and Tianjin Hospital of ITCWM Nankai Hospital (approval ID 2022026). Individual consent for this retrospective analysis was waived.

\section{Enrollment criteria}

Colorectal adenocarcinoma was confirmed through colonoscopy and bite biopsy, while intestinal cancer with synchronous liver metastases was confirmed through enhanced abdominal and pelvic CT, rectal MRI, serum alpha-fetoprotein (AFP) levels, and medical history. Patients' medical records, including genetic testing, were well documented, and complete follow-up records were kept. A professional and official MDT at the hospital level was responsible for developing the therapeutic regimens and supervising the entire process.

\section{Exclusion criteria}

Patients who had lung or craniocerebral metastases detected by enhanced chest CT or other methods were excluded. Moreover, patients were not eligible for the study if they could not tolerate systemic therapy because of their poor physical condition, discontinued or abandoned therapy in the course of the treatment, did not follow the treatment regimens determined by the MDT, or did not cooperate, had incomplete medical records, or were lost during the follow-up period. Patients who underwent acute emergency surgery for acute hemorrhage, perforation, and obstruction were also excluded.

\section{Treatment procedures}

The enrolled subjects were divided into the following two groups: (I) patients with lesions initially evaluated as resectable (the resectable group); and (II) patients with lesions initially evaluated as unresectable (the initially unresectable group). Phase I resection for primary lesions with liver metastases was performed on patients in the resectable group. Patients from the initially unresectable group were subdivided into an unresectable group and a potentially resectable group. Under the guidance of a MDT, regimens of conversion therapy, and targeted regimens of mFOLFOX and FOLFIRI were determined based on the results of clinical staging and genetic testing. The surgical resection of liver metastases, microwave ablation, radiofrequency ablation, and other therapies were performed on patients who had undergone successful conversion therapy. Systemic therapy was continued for patients (who indicated their willingness to participate) in whom conversion therapy was unsuccessful and whose lesions were still considered unresectable and non-ablative.

\section{Survival follow-up}

The clinical data of all the CRLM patients were included in each center's database of colorectal tumors. Followup appointments were carried out by the professional personnel of each center's team through the clinic service, telephone, or the WeChat messaging application. The reexamination results were reviewed, including results related to the physical examination, serum CEA level, AFP tumor marker test, analysis of peripheral blood cells, biochemical indexes, hepatic and renal function, CT scans of the chest and abdomen, and electronic colonoscopy. The survival data were recorded in detail. OS was defined as the time from diagnosis to death or discontinuation of follow-up. PFS was defined as the time from diagnosis to tumor progression or death. The last follow-up appointment was conducted on October 30, 2021.

\section{Statistical analysis}

The statistical analysis was conducted using SPSS 17.0 software. The measurement data are expressed as the mean \pm standard deviation, and a $t$-test was used to determine if the means of two data sets differed significantly. The enumeration data are represented by the number of cases, and a chi-square test was conducted. A logistic regression analysis was conducted to determine the factors affecting the success of conversion therapy. Kaplan-Meier survival curves were used for the monofactor survival analysis and a log-rank test was used to compare the differences in the survival rates. The significance of all statistical comparisons was set to $\mathrm{P}<0.05$.

\section{Results}

\section{General data and pathological characteristics of CRLM patients}

One hundred and seventy-five CRLM patients from three 


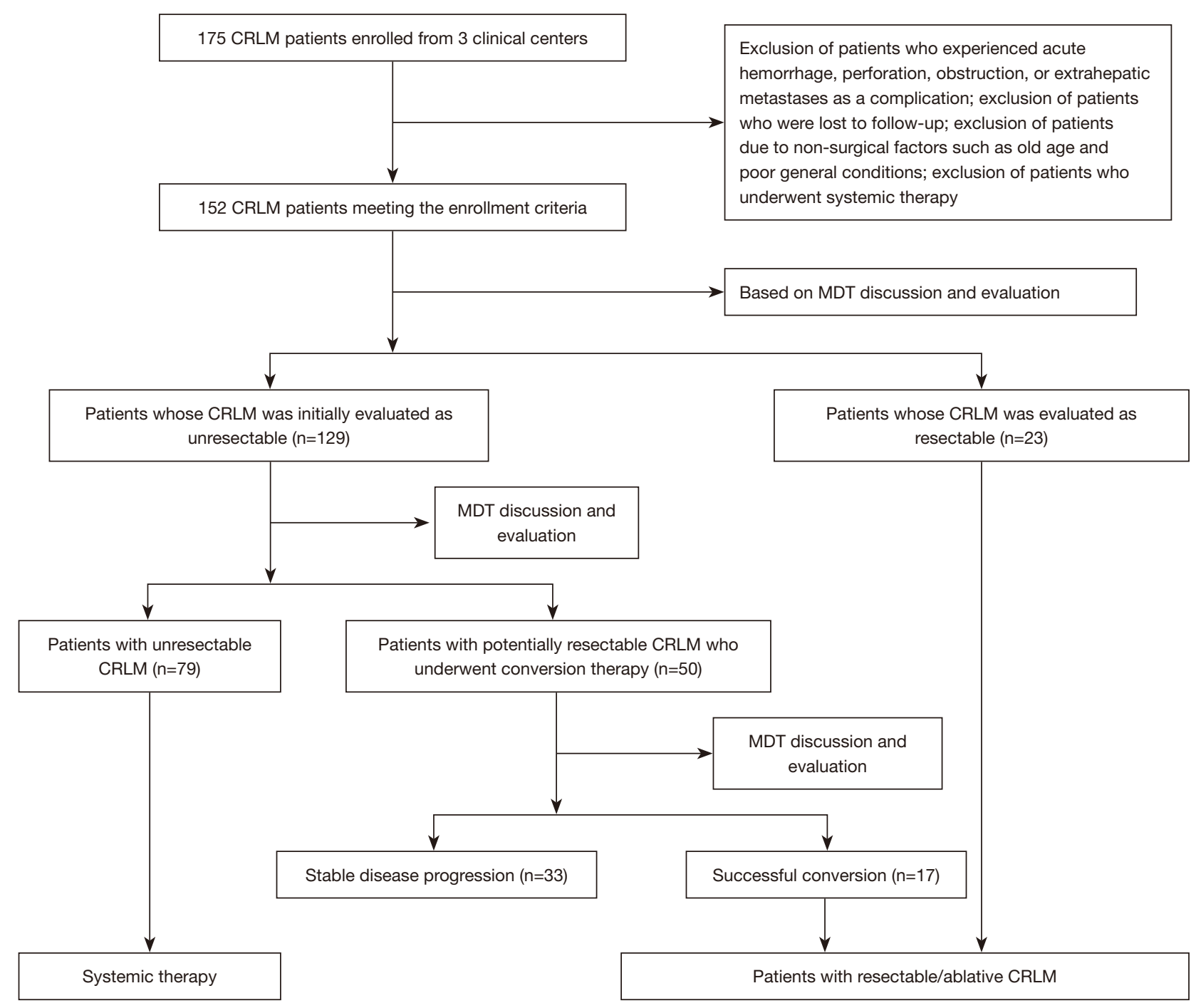

Figure 1 Enrollment flow chart of CRLM patients. CRLM, Colorectal Cancer Liver Metastasis; MDT, multidisciplinary.

medical centers were enrolled in this study. After excluding patients who underwent emergency surgery, developed extrahepatic metastases, or were lost during the follow-up period, 50 CRLM patients with potentially resectable liver metastases were enrolled for conversion therapy. Among them, 17 patients achieved NED status after undergoing therapy, 12 underwent surgical resection, 4 were treated with radiofrequency ablation, and 1 with microwave ablation (see Figures 1-3).

\section{Analysis of factors affecting conversion therapy in patients whose CRLM was initially evaluated as unresectable}

The general data and pathological characteristics of the patients, such as gender, age, the site of the primary lesion/ $\mathrm{s}$, the status of genetic testing, the serum CEA level, and the number of liver metastases, were analyzed. The results of the monofactor analysis indicated that the number and maximum diameter of liver metastases, the serum CEA level, the KRAS/NRAS and BRAF V600E mutation status, and the use of targeted drugs may be risk factors in determining whether NED status can be achieved through surgery/ablation following conversion therapy (see Table 1). The results of the logistic multifactor analysis demonstrated that the number of liver metastases had a significant effect on the efficacy of conversion therapy $(\mathrm{P}=0.048)$. The use of targeted drugs also affected the conversion therapy, but not significantly $(\mathrm{P}=0.063)$ (see Table 2). 

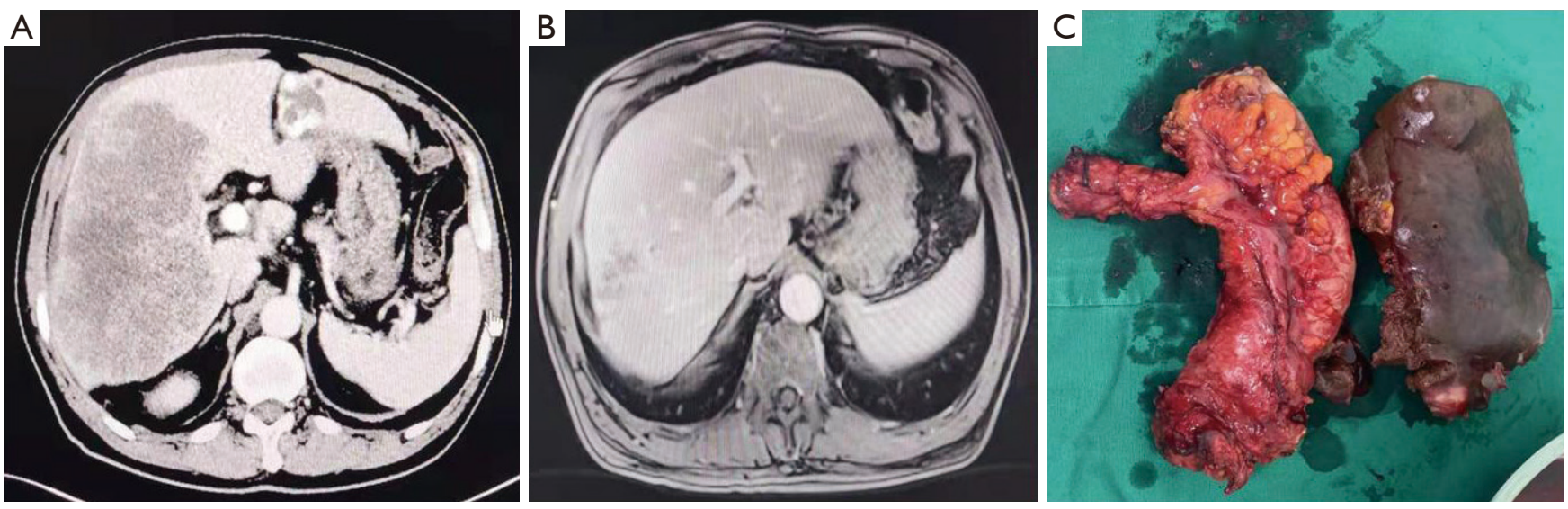

Figure 2 Cases of surgical resection of primary lesions and liver metastases after conversion therapy. (A) Abdominal CT of a patient whose CRLM was initially evaluated as potentially resectable; (B) abdominal CT of the patient at a follow-up visit after 8-cycle treatment with FOLFIRI + Cetuximab injections (biweekly); (C) primary lesions and liver metastases removed at the same resection. CT, computerized tomography; CRLM, Colorectal Cancer Liver Metastasis.
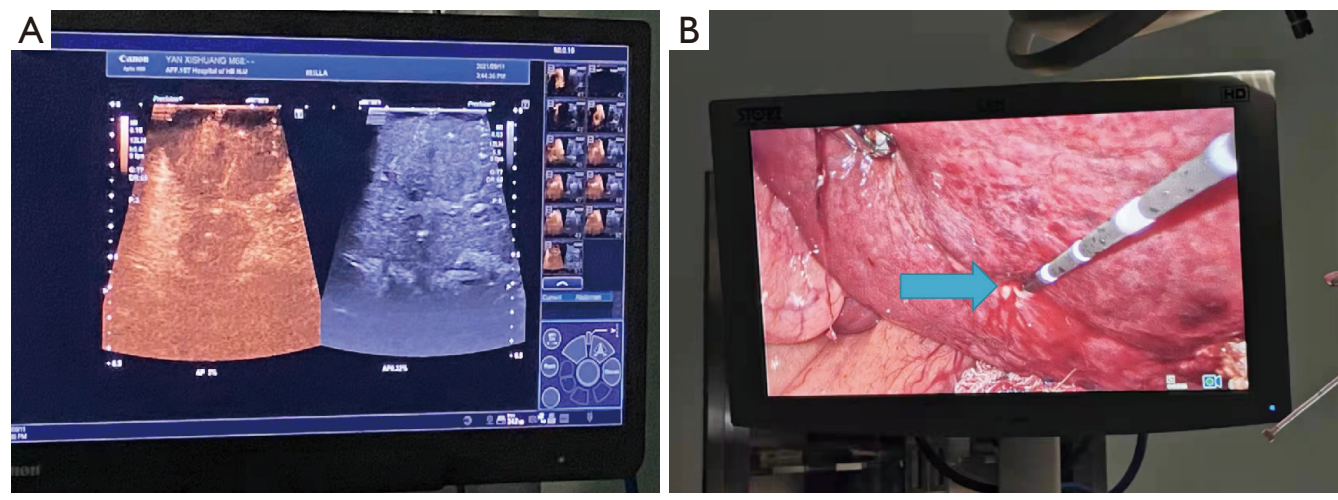

Figure 3 Intraoperative endoscopic ultrasound-guided microwave ablation of liver metastases. (A) Intraoperative ultrasound location and ablation of metastases; (B) laparoscopic ablation of metastases. The blue arrow means the location of liver metastases.

\section{Statistical analysis during the follow-up period}

The last follow-up appointment in this study was conducted on October 30, 2021. Overall, patients had an OS of 3-33 months with a median of 14 months. The OS of patients who successfully achieved NED through conversion therapy was $16-33$ months with a median of 22 months, while the OS of patients with inconspicuous/progressive lesions after undergoing conversion therapy was 3-20 months with a median of 11 months, which was significantly different between NED group and inconspicuous/progressive lesions group $(\mathrm{P}<0.0001)$ (see Figure 4). The OS of patients who underwent surgical resection was 16-32 months with a median of 23 months, while the OS of patients treated with ablation was 16-19 months with a median of 17 months, indicating statistical differences between surgical resection group and ablation group $(\mathrm{P}<0.05$; see Figure 5). Additionally, the PFS was 1-28 months with a median of 10 months. The PFS of patients who successfully achieved NED through conversion therapy was 13-28 months with a median of 18 months, while the PFS of patients with inconspicuous/progressive lesions after undergoing conversion therapy was 1-17 months with a median of 7 months, showing statistically significant differences between NED group and inconspicuous/progressive lesions group $(\mathrm{P}<0.0001$; see Figure 6). The PFS of patients who received a surgical resection was 14-28 months with a median of 22 months, while the PFS of patients treated with 
Table 1 General data and clinical and pathological characteristics of patients undergoing conversion therapy

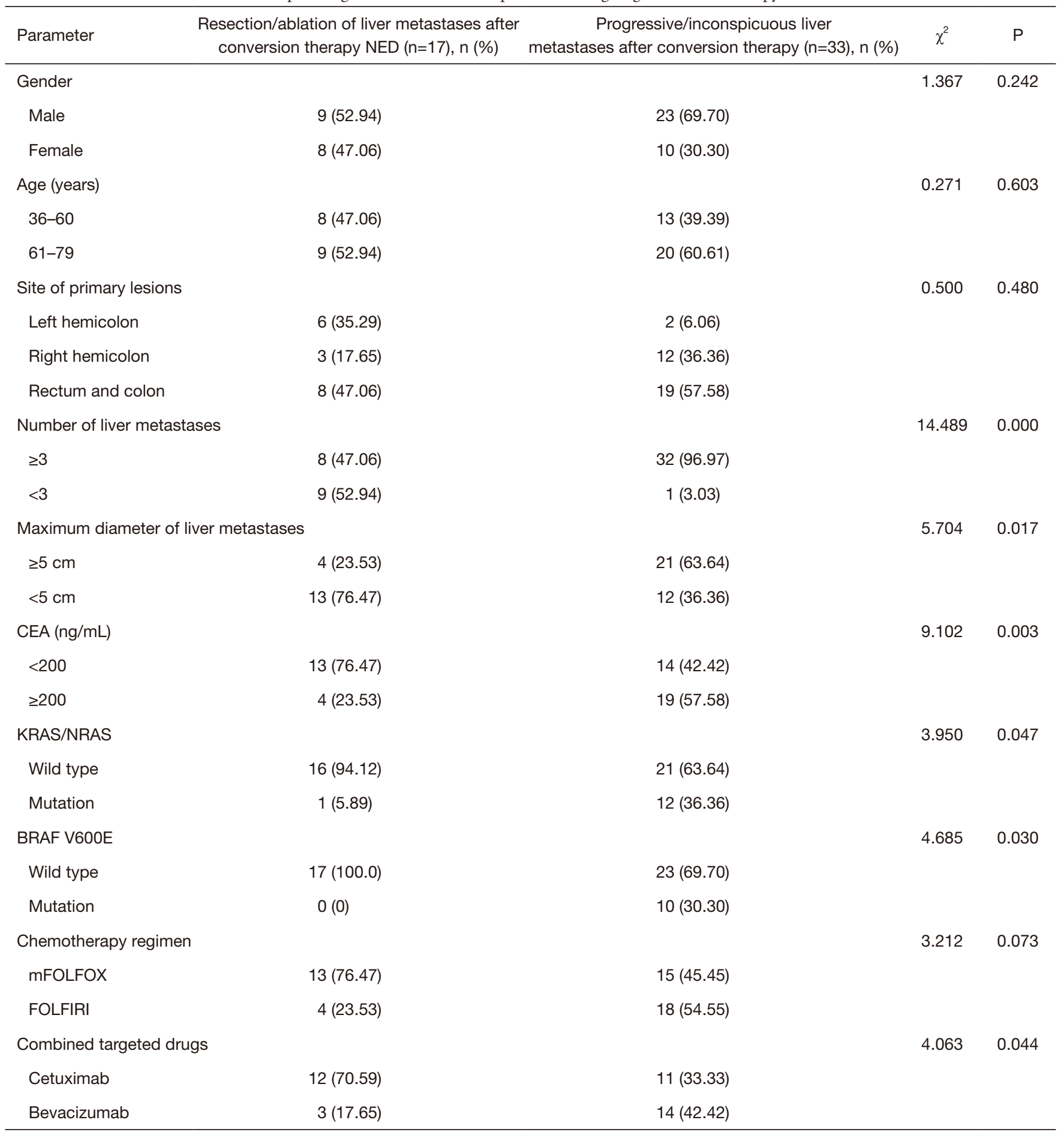

NED, no evidence of disease; CEA, Carcino-Embryonic Antigen. 
Table 2 Logistic regression analysis of multiple factors influencing the achievement of NED through conversion therapy

\begin{tabular}{lcccccc}
\hline Factor & $\beta$ & SE & Wald & P & OR & 95\% Cl \\
\hline Number of liver metastases & 2.371 & 1.197 & 3.923 & 0.048 & 10.704 & $1.025-111.765$ \\
Combined targeted drugs & 1.558 & 0.839 & 3.446 & 0.063 & 4.750 & $0.917-24.620$ \\
Constant & -2.520 & 1.183 & 4.538 & 0.033 & 0.080 & \\
\hline
\end{tabular}

NED, no evidence of disease.

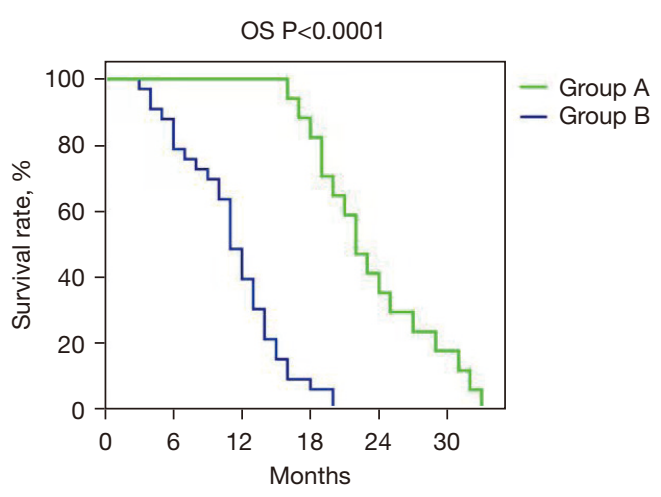

Figure 4 Kaplan-Meier analysis of the overall survival of the two patient groups (Group A) The patient group undergoing resection/ ablation of liver metastases after conversion therapy; (Group B) the patient group with progressive/inconspicuous liver metastases after conversion therapy. OS, overall survival.

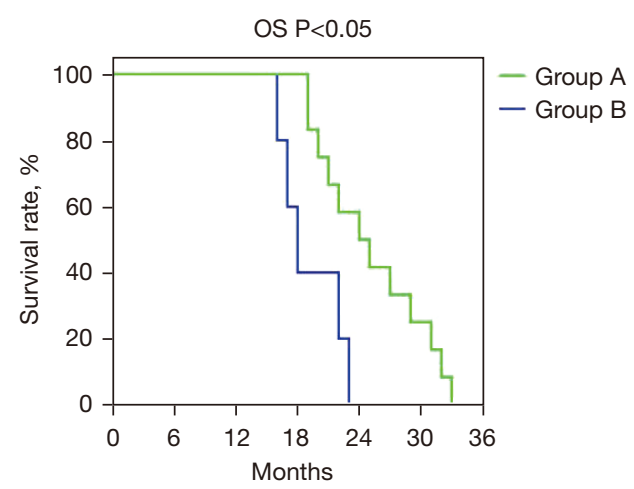

Figure 5 Kaplan-Meier analysis of the overall survival of the two patient groups. (Group A) The patient group undergoing surgical resection of liver metastases after conversion therapy; (Group B) the patient group undergoing (radiofrequency/microwave) ablation of liver metastases after conversion therapy. OS, overall survival.

ablation was $13-18$ months with a median of 14 months, which was significantly different between surgical resection group and ablation group $(\mathrm{P}<0.01$; see Figure 7).

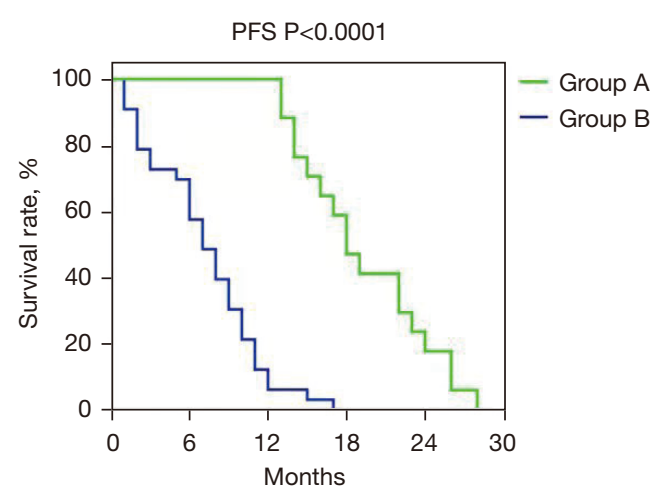

Figure 6 Kaplan-Meier analysis of the progression-free survival of the two patient groups. (Group A) The patient group undergoing resection/ablation of liver metastases after conversion therapy; (Group B) the patient group with progressive/inconspicuous liver metastases after conversion therapy. PFS, progression-free survival.

\section{Analysis of factors affecting the survival of patients whose CRLM was initially evaluated as unresectable}

Fifty CRLM patients were divided into two groups based on the median OS (i.e., OS $<14$ months and OS $\geq 14$ months). The results of the monofactor analysis revealed that the number and maximum diameter of liver metastases, the serum CEA level, and BRAF V600E mutations were factors affecting the OS of CRLM patients (see Table 3). More specifically, the results of the logistic multifactor analysis showed that BRAF V600E mutations alone were the primary determinant affecting OS (see Table 4).

\section{Discussion}

\section{Precise evaluation}

According to China's 2021 guidelines for CRLM diagnosis and comprehensive therapy, imaging tests (e.g., liver ultrasound and enhanced abdominal CT) should be performed in addition to rectal MRI, transrectal ultrasonography, CT evaluation, clinical staging, and 


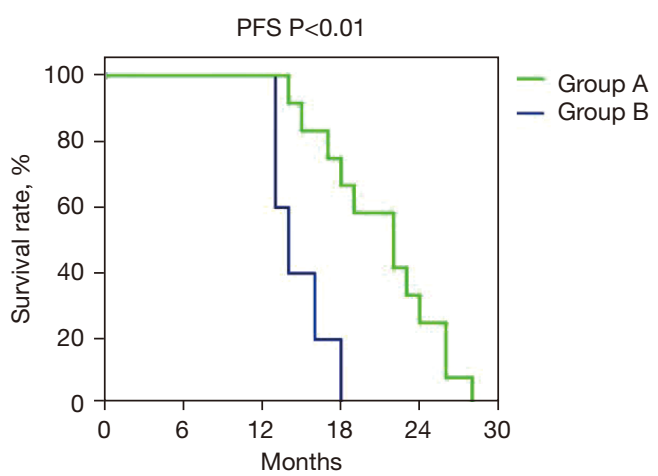

Figure 7 Kaplan-Meier analysis of the progression-free survival of the two patient groups. (Group A) The patient group undergoing resection of liver metastases after conversion therapy; (Group B) the patient group undergoing (radiofrequency/microwave) ablation of liver metastases after conversion therapy. PFS, progression-free survival.

routine tests of serum CEA, CA199, and other tumor markers to diagnose liver metastases in patients with pathologically confirmed colorectal cancer. For patients with suspected but unconfirmed liver metastases, additional methods, such as a serum AFP tumor marker test, liver ultrasound contrast, liver MRI plain scan, and enhanced tests should be conducted to undertake a comprehensive and precise evaluation of the sites, sizes, and the number of liver metastases, and determine the presence of synchronous metastases $(7,10)$. Additionally, CRLM patients should undergo genetic testing to analyze the mutational status of KRAS, NRAS, BRAF, Microsatellite Instability (MSI), and human epidermal growth factor receptor 2 (HER2).

\section{Development of therapeutic measures under the guidance of MDT}

MDT for colorectal tumors should comprise professional senior experts specializing in general (tumor and liver) surgery, medical oncology, gastroenterology, radiotherapy, pathology, nutrition, imaging, endoscopy, and psychotherapy and care (11). These specialists should work together to set therapeutic goals, develop treatment measures, and determine the time for subsequent visits based on each patient's medical condition, age, basic condition, psychological state, family, society status, and other influencing factors. The advantages are reflected in more accurate staging assessment, better connection of treatment, reduction of confusion and delay, full consideration of patients' quality of life and health economic and social effects, the best survival benefits, so as to achieve personalized and precise treatment (12-14).

In relation to the diagnosis of CRLM patients, the first step is to evaluate whether the liver metastases and the primary lesions can be resected in a single operation. This decision should be made based on the sites, number, and sizes of liver metastases. For patients whose CRLM is initially evaluated as unresectable, it is recommended that conversion therapy regimens be developed based on CRS scoring, the results of genetic testing, the sites of the primary lesions, and the patient's general condition, such as any underlying disease, nutrition, physical condition, and immune function, as all aspects of survival and the quality of life of patients who achieve NED status after treatment for liver metastases are considerably higher than those of patients who do not achieve it. For CRLM patients who may achieve NED status, conversion therapy is of particular importance. Additionally, the early shrinkage of metastatic lesions is an important indicator of favorable prognosis $(15,16)$.

MDT mode has been widely applied in clinical practice. Many international guidelines and expert recommendations emphasize the importance of multidisciplinary diagnosis and treatment, and also, MDT has been included in the guidelines in China, suggesting that all CRLM patients should participate in MDT diagnosis and treatment mode $(17,18)$. The overall prognosis of CRLM can be improved by making an individualized and most appropriate comprehensive treatment plan to maximize the benefit of patients.

\section{Treatment for resectable liver metastases of colorectal cancer}

For resectable liver metastases, preoperative chemotherapy could help shrink the tumor, test biological behavior, and reduce recurrence rate; but it may also cause liver injury and delay surgery. There is still controversy whether neoadjuvant chemotherapy should be performed and how to select patients from chemotherapy before surgery.

According to current domestic and foreign studies, the clinical benefit of direct surgery may outweigh the benefit of neoadjuvant tumor reduction in patients with obviously resectable liver metastases with overall good prognostic factors (CRS score $<2$ ). For patients with the number of metastases $>3$, the maximum diameter $\geq 5 \mathrm{~cm}$, the primary tumor with lymph node metastasis, serum CEA level increased and not receiving chemotherapy in the past 12 months, the clinical benefit of 
Table 3 Analysis of factors influencing survival in patients whose CRLM was initially evaluated as potentially resectable

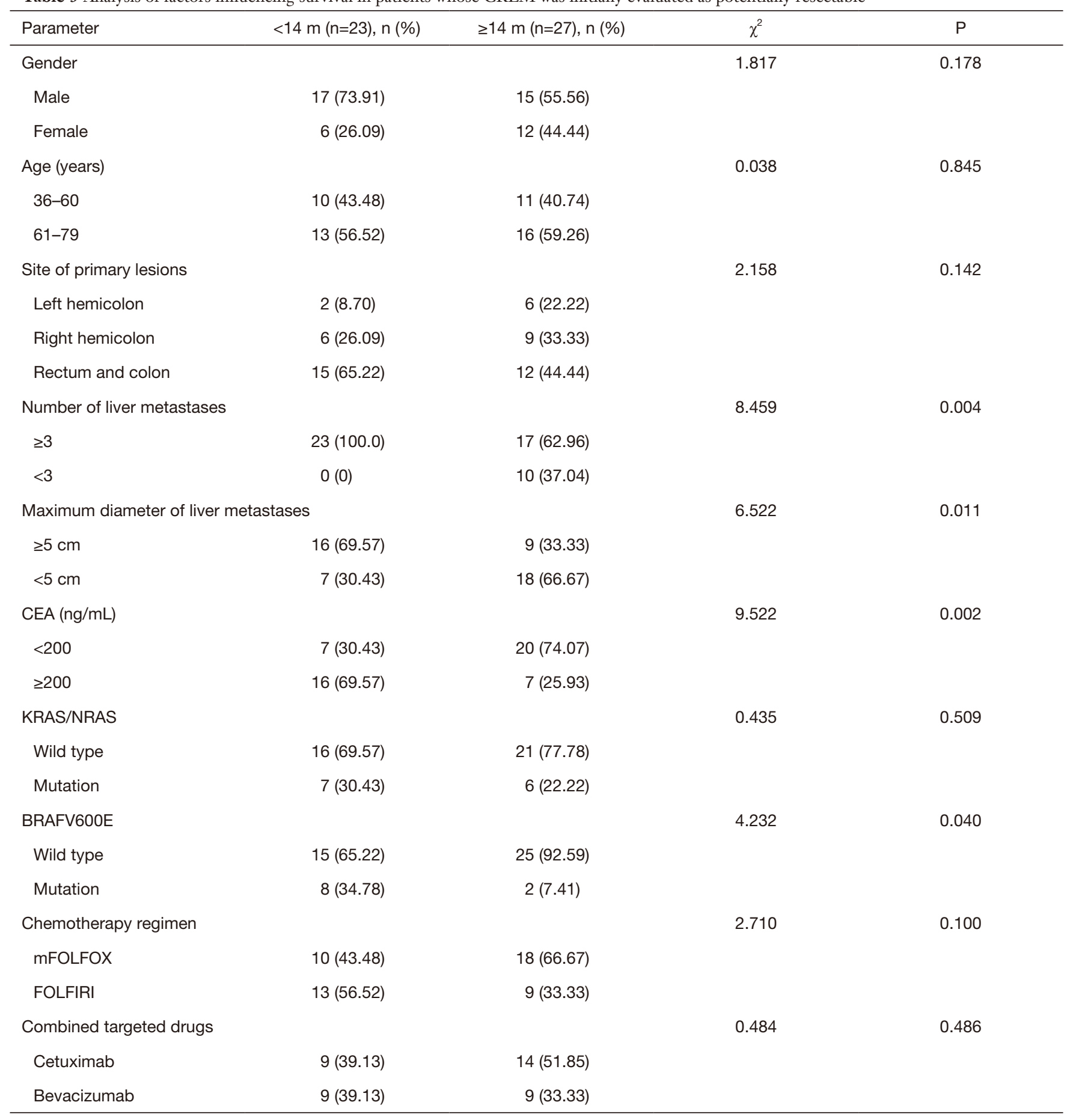

CRLM, Colorectal Cancer Liver Metastasis; CEA, Carcino-Embryonic Antigen. 
Table 4 Logistic multifactor analysis of patients whose CRLM was initially evaluated as potentially resectable

\begin{tabular}{lcccccc}
\hline Item & $\beta$ & $\mathrm{SE}$ & Wald & $\mathrm{P}$ & $\mathrm{OR}$ & $95 \% \mathrm{Cl}$ \\
\hline KARS/NARS & -3.908 & 2.061 & 3.594 & 0.058 & 0.020 & $0.000-1.141$ \\
BRAF V600E & -4.110 & 1.834 & 5.024 & 0.025 & 0.016 & $0.000-0.597$ \\
Constant & 50.076 & $13,900.835$ & 0.000 & 0.997 & 5.597 & \\
\hline
\end{tabular}

CRLM, Colorectal Cancer Liver Metastasis.

neoadjuvant chemotherapy may be greater than that with not neoadjuvant chemotherapy $(19,20)$.

\section{The challenges involved in providing comprehensive therapy to patients whose CRLM was initially evaluated as unresectable}

It is currently believed that complete surgical resection of liver metastases (CRLM) is the only possible treatment option for patients with colorectal cancer. For CRLM patients who were initially assessed as unresectable, the prognosis of patients who underwent resection after transformation was nearly identical to that of those who underwent the initial resection. Translational therapy is a method of transforming the initial unresectable foci into surgically resectable foci through preoperative systematic or local therapy for suitable patients to shrink the tumor. A number of studies have shown that the response rate of patients to preoperative chemotherapy drugs is significantly positively correlated with the conversion rate $(21,22)$. Therefore, according to the characteristics of patients with metastasis, individualized treatment with high response rate can improve the success rate of conversion therapy.

Multiple center studies have demonstrated that chemotherapy combined with targeted drugs improve the rate of conversion therapy $(23,24)$. The chemotherapy regimens commonly used for CRLM patients include FOLFIRI, FOLFOX, and FOLFOXIRI. The use of targeted drugs should be directed at the sites of primary lesions and the mutation status of KRAS and NRAS. In terms of primary lesions of the left hemicolon (including the rectum) with wild-type KRAS/NRAS, cetuximab is more effective against EGFR than VEGF given the OS and the objective remission rates. Conversely, for CRLM patients whose primary lesions are in the right hemicolon, cetuximab is less effective against EGFR than VEGF in terms of OS $(25,26)$. For CRLM patients with the BRAF V600E mutation, given the poor prognosis of these patients, FOLFOXIRI could be used in combination with cetuximab to inhibit VEGF (27-30).

For CRLM patients that undergo successful conversion therapy, the surgical resection of liver metastases is still the best approach. Apart from surgery, topical destruction therapies (e.g., radiofrequency ablation and microwave ablation) against some types of tumors can also lead to the thorough destruction of metastatic lesions $(31,32)$. Thus, the above-mentioned therapies can be considered for patients for whom surgical resection poses a substantial difficulty, for those for whom the postoperative residual liver volume is expected to be insufficient, and for those whose physical tolerance is poor, as this will enable more patients to achieve NED and improve the 5-year survival rate. Additionally, given the radiofrequency and microwave ablation therapies available for such patients, a MDT led by surgeons should be established to develop a comprehensive and individualized treatment plan based on MDT discussions and evaluations.

In this study, 50 patients from 3 regional medical centers whose CRLM was initially evaluated as potentially resectable met the inclusion criteria and were thus enrolled in the study. The pathological characteristics of these patients were analyzed and their survival data were collected. Our results demonstrated that $34 \%(17 / 50)$ of the patients achieved NED status through surgical resection and microwave/radiofrequency ablation after undergoing conversion therapy. The results of the monofactor analysis indicated that the influencing factors were the number and maximum diameter of liver metastases, serum CEA level, KRAS/NRAS mutation status, and the use of targeted drugs. More specifically, the results of the logistic multifactor analysis suggested that the number of liver metastases alone was the primary determinant, and the use of targeted drugs also had a certain effect. Due to the small sample, some indicators failed to meet the modeling requirements. In general, the OS and PFS of patients who achieved NED following successful conversion therapy was considerably higher than those of patients who failed to achieve NED after conversion therapy. Additionally, surgical resection led to better OS and PFS than treatment 
with microwave/radiofrequency ablation in CRLM patients. However, this finding might have been affected by the small number of cases included after stratification. Thus, further research based on a larger sample size and multicenter data needs to be conducted.

The results of Ma et al. from Peking University Cancer Hospital (33) demonstrated that radical surgical resection was successfully performed in $31.59 \%$ (24/76) of patients whose CRLM was initially evaluated as unresectable after they underwent conversion therapy which was slightly higher than $24 \%$ (12/50) of our study, and the median OS was significantly longer than that of the 52 patients whose conversion therapy was unsuccessful (20 and 15 months, respectively, $\mathrm{P}=0.034)$.

The results of the univariate analysis showed that the maximum diameter of liver metastases $<6 \mathrm{~cm}$ and a number of metastases $\leq 4$ were independent factors associated with successful conversion therapy. Nozawa et al. (34) examined 99 metastatic colorectal cancer patients who received chemotherapy, among whom, 23 patients (23.23\%) underwent radical surgical resection after conversion therapy, but found no significant difference in the long-term survival rate between these patients and the 112 patients with resectable phase-IV colorectal cancer who underwent surgery for the first time. Additionally, Bolhuis et al. (35) analyzed phase-II/III randomized trials published after 2008 on first-line systemic conversion therapy in patients or subgroups of patients with CRLM, and found that the mutational tumor status of KRAS/BRAF hampered conversion therapy in patients with unresectable CRLM (i.e., the $\mathrm{R} 0$ resection rate). To summarize, the results of the above-mentioned clinical studies are consistent with the findings of our study.

Further, a stratification analysis was conducted on the CRLM patients based on the median OS. The results of the monofactor analysis indicated that the number and maximum diameter of liver metastases, serum CEA level, and BRAF V600E mutational status affected the OS of CRLM patients, and that among these, the BRAF V600E mutation was the primary factor. However, concerns remain due to the failure of the relatively small number of parameters to meet the modeling requirements. Thus, a further demonstration is still needed based on a large sample of clinical data.

\section{Conclusions}

The use of advanced examination methods to correctly diagnose and treat CRLM is essential for patients whose CRLM is initially evaluated as unresectable. Developing precise therapeutic measures under the guidance of a MDT combines the advantages of different disciplines, and maximizes the chances of prolonging patient survival and improving their quality of life $(36,37)$. However, the treatment of these patients still faces substantial challenges. Undoubtedly, substantial benefits can be obtained by radical resection and ablation therapy in the case of some patients; however, the success rate of conversion therapy of about $30 \%$ is relatively low $(38,39)$. Improving this success rate of conversion therapy is the major difficulty facing current clinical research in the field of CRLM. Thus, more precise and effective regimens of conversion therapy need to be developed to improve the conversion rate and long-term survival of CRLM patients.

\section{Acknowledgments}

Funding: The study was supported by Special Tumor Research Project of National Cancer Center (No. NCC2017A23), the Hebei Funding Project for Highlevel Talents (No. A202101062), the Training Project for Excellent Clinical Medical Talents of Hebei Provincial Department of Finance (No. 361009), the 2015 Key Medical Science Project of Hebei Provincial Department of Science and Technology (No. 152777237), and the Science and Technology Mandatory Plan of Zhangiiakou, Hebei (No. 1712005D).

\section{Footnote}

Reporting Checklist: The authors have completed the STROBE reporting checklist. Available at https://apm. amegroups.com/article/view/10.21037/apm-22-87/rc

Data Sharing Statement: Available at https://apm.amegroups. com/article/view/10.21037/apm-22-87/dss

Conflicts of Interest: All authors have completed the ICMJE uniform disclosure form (available at https://apm. amegroups.com/article/view/10.21037/apm-22-87/coif). The authors have no conflicts of interest to declare.

Ethical Statement: The authors are accountable for all aspects of the work in ensuring that questions related to the accuracy or integrity of any part of the work are appropriately investigated and resolved. All procedures 
performed in this study involving human participants were in accordance with the Declaration of Helsinki (as revised in 2013). The study was approved by the ethics committees of The First Affiliated Hospital of Hebei North University (approval ID 2021013), Hebei Tumor Hospital (approval ID 2017kf386) and Tianjin Hospital of ITCWM Nankai Hospital (approval ID 2022026). Individual consent for this retrospective analysis was waived.

Open Access Statement: This is an Open Access article distributed in accordance with the Creative Commons Attribution-NonCommercial-NoDerivs 4.0 International License (CC BY-NC-ND 4.0), which permits the noncommercial replication and distribution of the article with the strict proviso that no changes or edits are made and the original work is properly cited (including links to both the formal publication through the relevant DOI and the license). See: https://creativecommons.org/licenses/by-nc-nd/4.0/.

\section{References}

1. Siegel RL, Miller KD, Goding Sauer A, et al. Colorectal cancer statistics, 2020. CA Cancer J Clin 2020;70:145-64.

2. Giannis D, Sideris G, Kakos CD, et al. The role of liver transplantation for colorectal liver metastases: A systematic review and pooled analysis. Transplant Rev (Orlando) 2020;34:100570.

3. Taniai N, Akimaru K, Yoshida H, et al. Surgical treatment for better prognosis of patients with liver metastases from colorectal cancer. Hepatogastroenterology 2007;54:1805-9.

4. Qin S, Liu GJ, Huang M, et al. The local efficacy and influencing factors of ultrasound-guided percutaneous microwave ablation in colorectal liver metastases: a review of a 4-year experience at a single center. Int J Hyperthermia 2019;36:36-43.

5. Norén A, Sandström P, Gunnarsdottir K, et al. Identification of Inequalities in the Selection of Liver Surgery for Colorectal Liver Metastases in Sweden. Scand J Surg 2018;107:294-301.

6. Margonis GA, Sergentanis TN, Ntanasis-Stathopoulos I, et al. Impact of Surgical Margin Width on Recurrence and Overall Survival Following R0 Hepatic Resection of Colorectal Metastases: A Systematic Review and Metaanalysis. Ann Surg 2018;267:1047-55.

7. Gastrointestinal Surgery Group, Surgical Society of Chinese Medical Association, Colorectal Surgery Group, Surgical Society of Chinese Medical Association, Colorectal Cancer Professional Committee, Chinese Anti-
Cancer Association, et al. Guideline for the diagnosis and comprehensive treatment of colorectal cancer liver metastasis (2018 edition). Chinese Journal of Digestive Surgery 2018;17:527-39.

8. Surgeons Branch of Chinese Medical Association, Gastrointestinal Surgery Group, Surgical Society of Chinese Medical Association, Colorectal Surgery Group, Surgical Society of Chinese Medical Association, et al. China guideline for diagnosis and comprehensive treatment of colorectal liver metastases (version 2020). Chinese Journal of Gastrointestinal Surgery 2021;24:1-13.

9. Weiser MR. AJCC 8th Edition: Colorectal Cancer. Ann Surg Oncol 2018;25:1454-5.

10. Adam R, de Gramont A, Figueras J, et al. Managing synchronous liver metastases from colorectal cancer: a multidisciplinary international consensus. Cancer Treat Rev 2015;41:729-41.

11. Xue J, Wu XL, Qu M, et al. Efficacy and safety analysis of neoadjuvant short-course radiotherapy combined with chemotherapy in low - and moderate-advanced rectal cancer under multidisciplinary collaboration: a single-center study. Journal of Practical Medicine 2021;36:1406-9.

12. Jung SM, Hong YS, Kim TW, et al. Impact of a Multidisciplinary Team Approach for Managing Advanced and Recurrent Colorectal Cancer. World J Surg 2018;42:2227-33.

13. Schiergens TS, von Einem J, Thomas MN, et al. Multidisciplinary treatment of colorectal liver metastases. Minerva Med 2017;108:527-46.

14. Wanis KN, Pineda-Solis K, Tun-Abraham ME, et al. Management of colorectal cancer with synchronous liver metastases: impact of multidisciplinary case conference review. Hepatobiliary Surg Nutr 2017;6:162-9.

15. Piessevaux $H$, Buyse $M$, Schlichting $M$, et al. Use of early tumor shrinkage to predict long-term outcome in metastatic colorectal cancer treated with cetuximab. J Clin Oncol 2013;31:3764-75.

16. Okazaki S, Ishikawa T, Uetake H. Perioperative Chemotherapies for Patients with Colorectal Liver Metastasis. Gan To Kagaku Ryoho 2020;47:574-7.

17. Abukar AA, Ramsanahie A, Martin-Lumbard K, et al. Availability and feasibility of structured, routine collection of comorbidity data in a colorectal cancer multidisciplinary team (MDT) setting. Int J Colorectal Dis 2018;33:1057-61.

18. Li X, Chen Q, Bi X, et al. Effects of multidisciplinary team on the outcomes of colorectal cancer patients with liver metastases Ann Palliat Med 2020;9:2741-8. 
19. Chen G, Li YH, Zhang RX, et al. Rethinking of neoadjuvant therapy for patients with initially resectable colorectal cancer liver metastases. Chinese Journal of Hepatobiliary Surgery 2020;26:488-92.

20. Wang LJ, Wang HW, Jin KM, et al. Comparative study on prognosis of neoadjuvant chemotherapy followed by hepatic surgery versus upfront surgery in patients with synchronous colorectal liver metastasis. Chinese Journal of Gastrointestinal Surgery 2021;24:248-55.

21. Maeda Y, Shinohara T, Nagatsu A, et al. Long-Term Outcomes of Conversion Hepatectomy for Initially Unresectable Colorectal Liver Metastases. Ann Surg Oncol 2016;23:S242-8.

22. Iida H, Kaibori M, Wada H, et al. Prognostic factors of hepatectomy in initially unresectable colorectal liver metastasis: Indication for conversion therapy. Mol Clin Oncol 2018;9:545-52.

23. Pernot S, Artru P, Mithieux F, et al. Complete pathological response of unresectable liver metastases from colorectal cancer after trans-arterial chemoembolization with drugeluting beads loaded with irinotecan (DEBIRI) and concomitant systemic FOLFOX: A case report from the FFCD 1201 trial. Clin Res Hepatol Gastroenterol 2015;39:e73-7.

24. Alberts SR, Horvath WL, Sternfeld WC, et al. Oxaliplatin, fluorouracil, and leucovorin for patients with unresectable liver-only metastases from colorectal cancer: a North Central Cancer Treatment Group phase II study. J Clin Oncol 2005;23:9243-9.

25. Ye LC, Liu TS, Ren L, et al. Randomized controlled trial of cetuximab plus chemotherapy for patients with KRAS wild-type unresectable colorectal liver-limited metastases. J Clin Oncol 2013;31:1931-8.

26. Folprecht G, Gruenberger T, Bechstein WO, et al. Tumour response and secondary resectability of colorectal liver metastases following neoadjuvant chemotherapy with cetuximab: the CELIM randomised phase 2 trial. Lancet Oncol 2010;11:38-47.

27. Karapetis CS, Jonker D, Daneshmand M, et al. PIK3CA, BRAF, and PTEN status and benefit from cetuximab in the treatment of advanced colorectal cancer--results from NCIC CTG/AGITG CO.17. Clin Cancer Res 2014;20:744-53.

28. Ghiringhelli F, Vincent J, Bengrine L, et al. Hepatic arterial chemotherapy with raltitrexed and oxaliplatin versus standard chemotherapy in unresectable liver metastases from colorectal cancer after conventional chemotherapy failure (HEARTO): a randomized phase-II study. J Cancer Res Clin Oncol 2019;145:2357-63.

29. Ye LC, Wei Y, Zhu DX, et al. Impact of early tumor shrinkage on clinical outcome in wild-type-KRAS colorectal liver metastases treated with cetuximab. J Gastroenterol Hepatol 2015;30:674-9.

30. Loupakis F, Cremolini C, Salvatore L, et al. FOLFOXIRI plus bevacizumab as first-line treatment in BRAF mutant metastatic colorectal cancer. Eur J Cancer 2014;50:57-63.

31. Facciorusso A, Di Maso M, Muscatiello N. Microwave ablation versus radiofrequency ablation for the treatment of hepatocellular carcinoma: A systematic review and meta-analysis. Int J Hyperthermia 2016;32:339-44.

32. Xu Z, Xie H, Zhou L, et al. The Combination Strategy of Transarterial Chemoembolization and Radiofrequency Ablation or Microwave Ablation against Hepatocellular Carcinoma. Anal Cell Pathol (Amst) 2019;2019:8619096.

33. Ma R, Li T. Conversion therapy combined with individualized surgical treatment strategy improves survival in patients with colorectal cancer liver metastases. Int J Clin Exp Pathol 2021;14:314-21.

34. Nozawa H, Ishihara S, Kawai K, et al. Conversion to Resection in Patients Receiving Systemic Chemotherapy for Unresectable and/or Metastatic Colorectal CancerPredictive Factors and Prognosis. Clin Colorectal Cancer 2018;17:e91-7.

35. Bolhuis K, Kos M, van Oijen MGH, et al. Conversion strategies with chemotherapy plus targeted agents for colorectal cancer liver-only metastases: A systematic review. Eur J Cancer 2020;141:225-38.

36. Xu D, Xing BC. Rethinking of the conversion therapy in colorectal cancer patients with liver metastasis. Chinese Journal of Oncology 2019;41:81-5.

37. Zhang YY, Chen SW, Wang PY, et al. Research progress of conversion therapy in colorectal cancer liver metastases. Chinese Journal of Gastrointestinal Surgery 2021;24:85-93.

38. Villard C, Habib M, Nordenvall C, et al. Conversion therapy in patients with colorectal liver metastases. Eur J Surg Oncol 2021;47:2038-45.

39. Zhai S, Sun X, Du L, et al. Role of Surgical Approach to Synchronous Colorectal Liver Metastases: A Retrospective Analysis. Cancer Manag Res 2021;13:3699-711.

Cite this article as: Han L, Xue J, Wu X, Liang G, Wu Y, Guo F, Gao S, Sun G. Multidisciplinary approach to the diagnosis and treatment of patients with potentially resectable colorectal cancer liver metastasis: results of a multicenter study. Ann Palliat Med 2022;11(2):717-729. doi: 10.21037/apm-22-87 